\title{
Two causes of palpitations, detected by photoplethysmog- raphy on a mobile phone
}

\author{
W. Gielen · M. Gielen
}

Published online: 27 November 2018

(C) The Author(s) 2018

A 54-year-old woman, treated for hypertension, visits her general practitioner with symptoms of recurrent palpitations. Earlier Holter monitoring has been normal except for premature atrial contractions. Her symptoms occur spontaneously and are not stress-related. Physical examination and ECG were without ab-
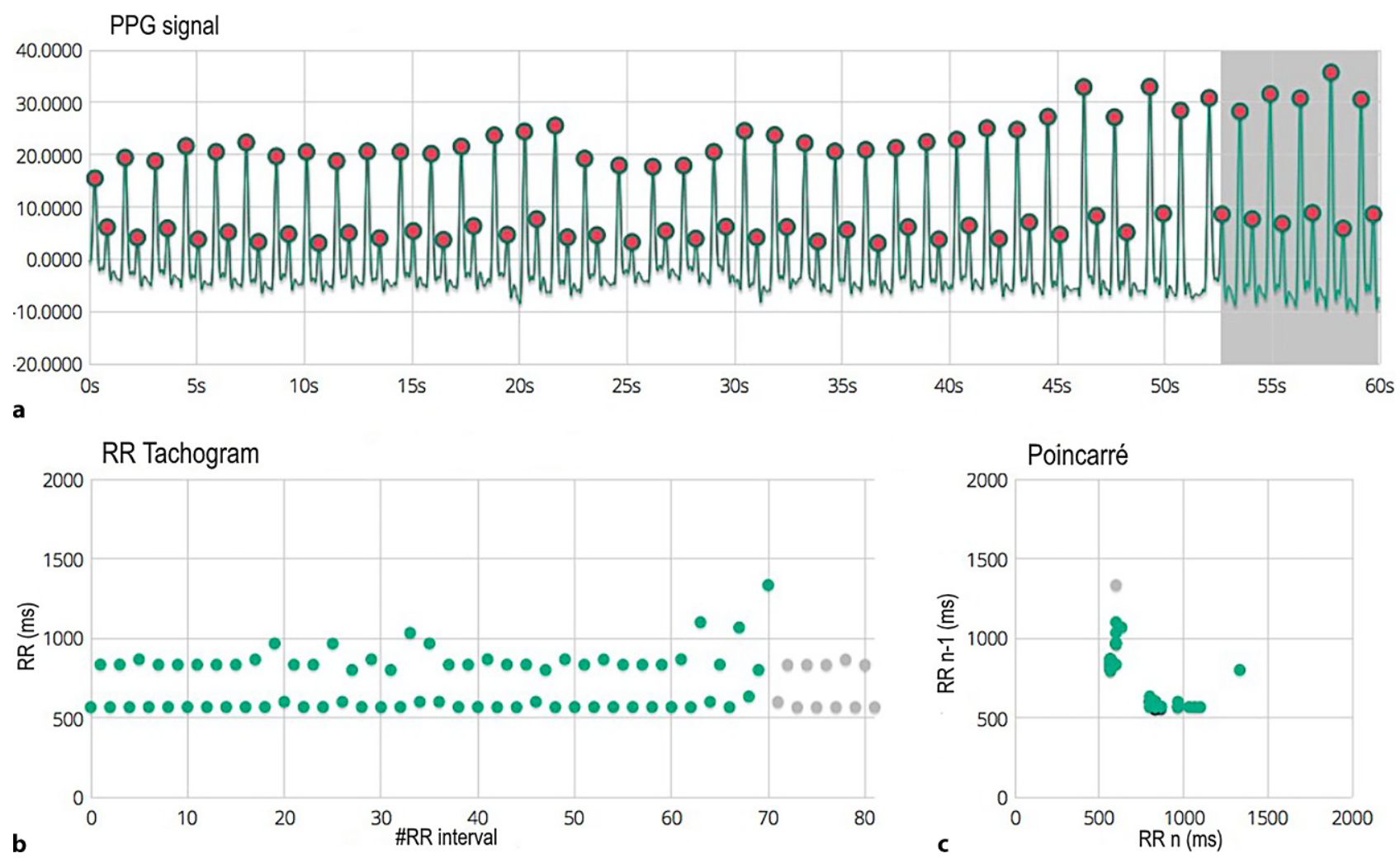

Fig. 1 Photoplethysmogram (PPG) recording. a 60s PPG signal measured at the patient's fingertip with the phone camera. b RR tachogram which represents the distance between

the RR intervals in milliseconds. c The Poincaré plot shows how the present RR interval is related to the previous one

\section{W. Gielen $(\bowtie) \cdot$ M. Gielen}

Silkeborg Regional Hospital, Silkeborg, Denmark

willem.gielen@rm.dk 

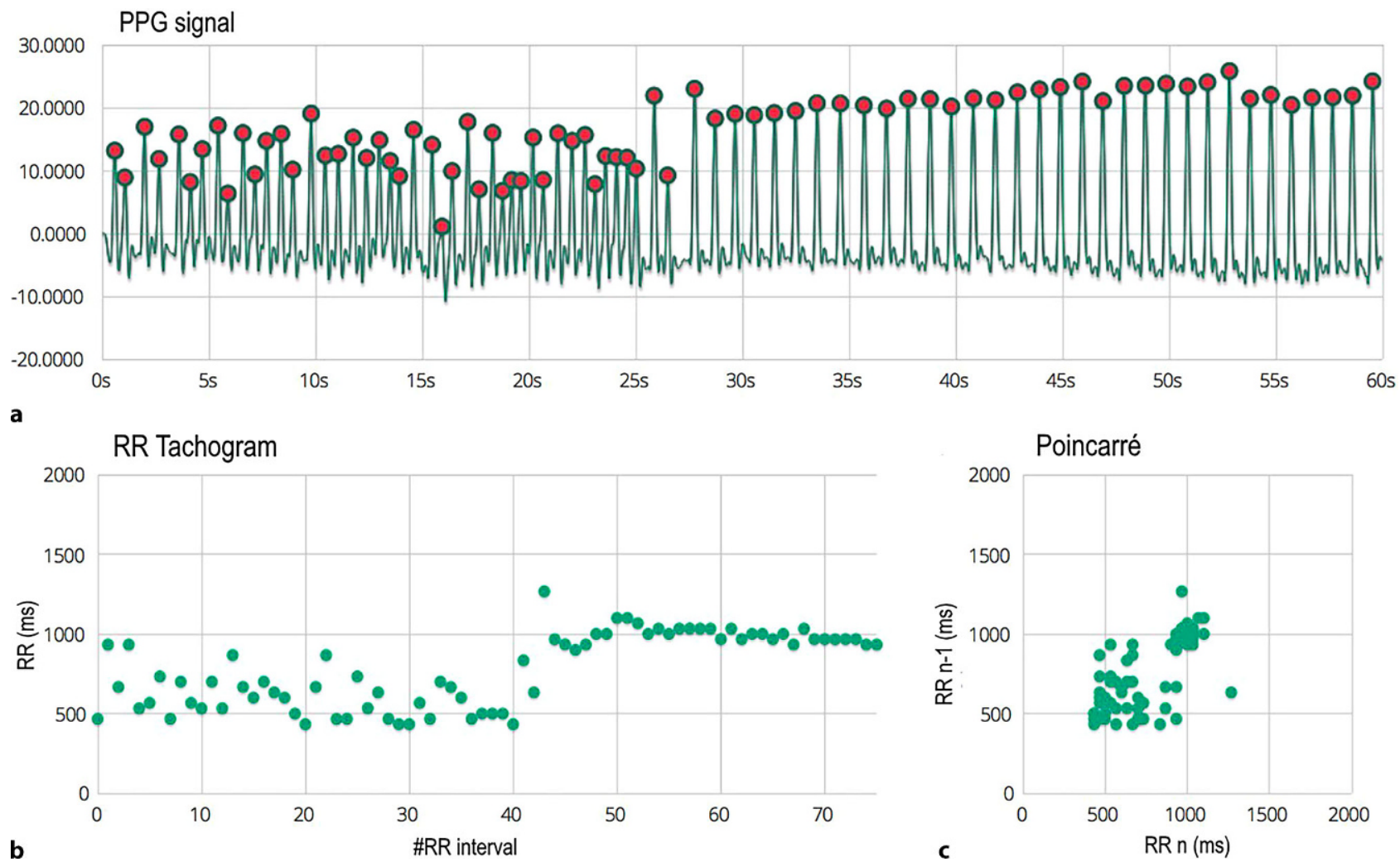

Fig. 2 Photoplethysmogram (PPG) recording. a 60s PPG signal measured at the patient's fingertip with the phone camera. b RR tachogram which represents the distance between

normalities. To document her symptoms over a longer period of time, her general practitioner prescribed the FibriCheck app for the duration of a month, which she could download onto her mobile phone. In case of any symptoms, she had to put her finger on her phone's camera. This allowed FibriCheck to record the rhythm for a duration of $60 \mathrm{~s}$ using photoplethysmography (PPG), a simple optical technique used to detect volumetric changes in the blood in the peripheral circulation (Figs. 1 and 2; [1-3]). The results were automatically analysed by the algorithm, then sent to, analysed and validated by a local cardiologist. With symptoms three kinds of rhythms were observed.

Which rhythms can be recognised on these PPG recordings?

\section{Answer}

You will find the answer elsewhere in this issue.

the RR intervals in milliseconds. c The Poincaré plot shows how the present RR interval is related to the previous one

Open Access This article is distributed under the terms of the Creative Commons Attribution 4.0 International License (http://creativecommons.org/licenses/by/4.0/), which permits unrestricted use, distribution, and reproduction in any medium, provided you give appropriate credit to the original author(s) and the source, provide a link to the Creative Commons license, and indicate if changes were made.

\section{References}

1. Mortelmans C, Van Haelst R, Van Der Auwera J, Grieten L, Vandervoort P, Vaes B. 180 Validation of a new smartphone application for the diagnosis of atrial fibrillation in primary care, EP Europace, 2017;19(Suppl3):iiil6. https://doi.org/ 10.1093/ehjci/eux136.008.

2. Cheung CC, Krahn AD, Andrade JG. The emerging role of wearable technologies in arrhythmia detection. Can J Cardiol. 2018; https://doi.org/10.1016/j.cjca.2018.05.003.

3. Tang S-C, Huang P-W, Hung C-S, et al. Identification of atrial fibrillation by quantitative analyses of fingertip photoplethysmogram. Sci Rep. 2017;7:45644. 